\title{
Single-layer continuous suture contributes to the reduction of surgical complications in digestive tract anastomosis involving special anatomical locations
}

\author{
GUO-CAI LI, YU-CHUN ZHANG, YONG XU, FANG-CHENG ZHANG, \\ WEI-HUA HUANG, JIAN-QING XU and QING-JIU MA \\ Department of General Surgery, Xi'an Gaoxin Hospital, Xi'an Jiaotong University, Xi'an, Shaanxi 710075, P.R. China
}

Received June 19, 2013; Accepted October 25, 2013

DOI: $10.3892 /$ mco.2013.215

\begin{abstract}
The key point of digestive cancer surgery is reconstruction and anastomosis of the digestive tract. Traditional anastomoses involve double-layer interrupted suturing, manually or using a surgical stapler. In special anatomical locations, however, suturing may become increasingly difficult and the complication rate increases accordingly. In this study, we aimed to investigate the feasibility and safety of a new manual suturing method, the single-layer continuous suture in the posterior wall of the anastomosis. Between January, 2007 and August, 2012, 101 patients with digestive cancer underwent surgery in Xi'an Gaoxin Hospital. Of those patients, 27 underwent surgery with the new manual method and the remaining 74 underwent surgery using traditional methods of anastomosis of the digestive tract. Surgical time, intraoperative blood loss, drainage duration, complications, blood tests, postoperative quality of life (QOL) and overall expenditure were recorded and analyzed. No significant differences were observed in surgical time, intraoperative blood loss, temperature, blood tests and postoperative QOL between the two groups. However, compared with the control group, the new manual suture group exhibited a lower surgical complication rate (7.40 vs. $31.08 \% ; \mathrm{P}=0.018$ ), lower blood transfusion volume (274.07 \pm 419.33 vs. $646.67 \pm 1,146.06 \mathrm{ml} ; \mathrm{P}=0.053)$, shorter postoperative hospital stay ( $14.60 \pm 4.19$ vs. $17.60 \pm 6.29$ days; $\mathrm{P}=0.038)$ and lower overall expenditure $(3,509.85 \pm 768.68$ vs. $6,141.83 \pm 308.90$ renminbi; $\mathrm{P}=0.001$ ). Our results suggested that single-layer continuous suturing for the anastomosis of the digestive tract is feasible and safe and may contribute to the reduction of surgical complications and overall expenditure.
\end{abstract}

Correspondence to: Professor Qing-Jiu Ma, Department of General Surgery, Xi'an Gaoxin Hospital, Xi'an Jiaotong University, 16 South Tuanjie Road, Xi'an, Shaanxi 710075, P.R. China E-mail: maqjyue@sina.com

Key words: anastomosis, gastric cancer, rectal cancer, single-layer, continuous suture

\section{Introduction}

Gastric cancer is currently a leading cause of cancer-related mortality. Despite the overall decrease in morbidity over the last few years, gastric cancer remains the second leading cause of cancer-related mortality worldwide and the first in China (1). The morbidity of colorectal cancer has increased, making it the third most common malignancy in China (2). The key point of surgery for these cancers is reconstruction and anastomosis of the digestive tract (3). The methods of anastomosis are closely associated with the outcome of surgery, postoperative quality of life (QOL) and complication rate.

Traditional manual suturing and surgical staples are commonly used in clinical practice. The main anastomotic complications include leakage, stricture and infection. It was reported that, in esophagogastric anastomosis, the incidence rate of leakage and stricture was $0-21.9$ and $0-25.8 \%$, respectively, with manual suturing and $0-19.5$ and $0-32.8 \%$, respectively, with surgical stapling (4-8). Furthermore, the incidence of leakage was reported to be $2.6-17 \%$ in low anterior resection for rectal cancer with the double-stapling technique (9-12).

Traditional manual anastomosis with double-layer interrupted suture is a complicated procedure, depending largely on the skill of the surgeon. Furthermore, in certain surgeries, such as esophagogastrostomy and colorectostomy, the anastomosis may be more difficult to perform, owing to the special anatomical location, and the complication rate increases accordingly.

Stapling devices are used for suturing in difficult anastomotic locations. With the use of staplers, an increasing number of proximal gastric cancers have become resectable through the abdominal cavity and the number of anal-preserving surgeries for rectal cancer is also on the increase. Stapling devices may decrease surgery time, surgical trauma and anastomotic complications (13-16). However, improper handling of the stapler may lead to a partial tear of the anastomosis and leakage $(17,18)$, whereas irregular suturing of the mucosa may lead to hyperplasia, granulation tissue and scar formation, which may cause a stricture $(19,20)$. Furthermore, there was no reported difference in the morbidity and mortality rates between the hand-sewn and stapled techniques $(21,22)$. Additionally, stapling has been criticized as it confers an increase in the overall expediture and, 
in certain cases, such as preoperative obstruction of the digestive tract, mucosal edema, increased thickness of the muscle layer and healing dysfunction, the use of staplers is restricted. Therefore, a simple and convenient method for performing anastomoses in special anatomical locations is required.

Single-layer continuous suture is a method commonly used for vessel anastomosis in deep locations. The tightness and safety of the single-layer suture has been proven in anastomoses of the digestive tract $(23,24)$. However, the combination of single-layer and continuous suturing in anastomosis is rarely reported.

In this study, we aimed to investigate a new manual method for difficult anastomotic locations, which is the single-layer continuous suture in the posterior wall of the anastomosis. We consider this method to be feasible and safe and it may help simplify procedures that are considered difficult due to special anatomical locations, solve the problem of instrument shortage for instrumental anastomosis and reduce expenditure.

\section{Materials and methods}

Patients. Between January, 2007 and August, 2012, 350 consecutive patients with gastric or colorectal cancer underwent open surgery in the Department of General Surgery, Xi'an Jiaotong University, China. For this prospective cohort study, the patient inclusion criteria were as follows: i) adult patients ( $>18$ years); ii) patients with gastric cancer who underwent proximal or total gastric resection with esophagogastric or esophagojejunal anastomosis, whereas those with distal gastric resection or palliative resection were excluded; and iii) patients with colorectal cancer who underwent low anterior resection of the rectum or left-side colostomy followed by colon-to-rectum anastomosis, whereas those undergoing local excision, Hartmann procedure, Miles procedure, palliative treatment or right-side colostomy were excluded.

Of the 101 patients who met the inclusion criteria and were enrolled in this cohort study, 65 underwent esophagogastric anastomosis and 36 underwent colorectal anastomosis. The patients were grouped according to the treatment received as follows: i) new manual suture, 27 patients; ii) traditional manual suture, 65 patients; and iii) stapling, 9 patients. The clinical and pathological details of all the cases were carefully recorded. The TNM clinical and pathological staging classification was used for gastric cancer and Dukes' staging classification was used for rectal cancer.

This study was approved by the Ethics Committee of the Xi'an Jiaotong University. All patients received verbal and written information regarding the study and provided informed consent prior to their enrollment.

Demographic and preoperative data. Demographic data, including gender, age, smoking status, alcohol consumption and disease history were collected. Preoperative data, including routine hematological and biochemical tests and $\mathrm{X}$-rays were collected to enable a subsequent analysis of the comparability of the groups.

\section{Procedures}

Gastroesophageal anastomosis. All surgeries were performed under general anaesthesia. Bowel preparation and antibiotic prophylaxis were standardized. An upper midline abdominal incision was performed, followed by spleen-preserving D2 lymphadenectomy.

The staple suturing was performed as follows: the distal esophagus was transected and a purse-string suture was placed on the proximal end. Subsequently, the anvil of a 28-mm diameter circular stapler was introduced into the distal esophageal end, the central shaft of the gun was thrust through the anterior wall of the distal greater curvature and was assembled with the anvil (outer ring). An end-to-side anastomosis was created with the button, the gun was withdrawn and the residual gastric end was closed.

In the traditional manual suture group, the lesser curvature lateral to the gastric end was closed and the greater curvature was prepared for end-to-end anastomosis. The double-layer interrupted suture with silk thread was used in the posterior and anterior wall of the anastomosis (total layer suture combined with embedding of the serosal and muscle layer).

The new manual suture was performed as follows: the single-layer continuous suture with 4-0 prolene thread (Ethicon, Inc., Somerville, NJ, USA) was used in the posterior wall of the anastomosis and the double-layer interrupted suture with silk thread was used in the anterior wall. Abdominal drains were inserted and the abdominal incision was closed to complete the surgery.

Colorectal anastomosis. Bowel preparation consisted of fluids, oral cathartics, antibotics and enemas for 3 days prior to the surgery. The laparotomy was performed through a midline incision. All patients underwent standard rectal dissection with pelvic peritonectomy. A circumferential dissection of the rectum was performed up to the level of $6 \mathrm{~cm}$ from the dentate line. The proximal colon was progressively released to ensure a tension-free anastomosis. On completion of the resection, an anastomosis was performed. The posterior and anterior wall were sutured with a double-layer interrupted suture with silk thread in the traditional manual group.

The new manual suture was performed as follows: the posterior wall of the anastomosis was sutured using the single-layer continuous suture with a 4-0 prolene thread and the anterior wall was sutured with the traditional manual technique.

The staple suture was performed with a 32-mm diameter circular stapler (Johnson \& Johnson, New Brunswick, NJ, USA), with the anvil placed on the distal end of the colon. The central shaft of the gun was introduced into the residual rectum via the anal canal and assembled with the anvil. An end-to-end colon-to-rectum anastomosis was created with the button. The abdominal incision was closed in layers.

Postoperative observations. The volume of the drainage fluid was recorded at 1,2, 3 and 7 days following the surgery. The surgical time was measured from the first skin incision to the last suture placement for all the techniques. The recovery time of gastrointestinal function, intraoperative blood loss, total amount of blood transfusion and the highest postoperative temperature were recorded. The surgical specimens, together with any separately harvested lymph nodes, were placed in formalin and transported to the laboratory for pathological examination. The histological subtype and pathological stage were recorded using the Union for International Cancer 
Table I. Characteristics of patients in new manual and traditional method groups.

\begin{tabular}{|c|c|c|c|c|c|c|}
\hline \multirow[b]{2}{*}{ Characteristics } & \multicolumn{3}{|c|}{ Gastric cancer } & \multicolumn{3}{|c|}{ Rectal cancer } \\
\hline & New manual & Traditional & P-value & New manual & Traditional & P-value \\
\hline Male/female & $15 / 4$ & $31 / 15$ & 0.550 & $3 / 5$ & $14 / 14$ & 0.695 \\
\hline Age, years $($ mean $\pm S D)$ & $58.89 \pm 11.31$ & $63.50 \pm 11.68$ & 0.207 & $48.14 \pm 25.61$ & $61.70 \pm 15.44$ & 0.133 \\
\hline Tumor size, cm & $4.48 \pm 2.01$ & $5.89 \pm 3.25$ & 0.165 & $5.17 \pm 2.79$ & $5.67 \pm 2.33$ & 0.668 \\
\hline \multicolumn{7}{|l|}{ Lymph node involvement } \\
\hline Negative & 5 & 10 & 0.753 & 5 & 15 & 0.709 \\
\hline Positive & 14 & 35 & - & 3 & 13 & - \\
\hline Unknown & 0 & 1 & - & 0 & 0 & - \\
\hline \multicolumn{7}{|l|}{ Grade of differentiation } \\
\hline High & 2 & 4 & 0.755 & 4 & 9 & 0.635 \\
\hline Moderate & 8 & 22 & - & 4 & 15 & - \\
\hline Poor & 9 & 18 & - & 0 & 2 & - \\
\hline Unknown & 0 & 2 & - & 0 & 2 & - \\
\hline \multicolumn{7}{|l|}{ Pathological type } \\
\hline Adenocarcinoma & 12 & 28 & 0.990 & 5 & 22 & 0.384 \\
\hline Non-adenocarcinoma & 7 & 18 & - & 3 & 6 & - \\
\hline \multicolumn{7}{|l|}{ TNM stage } \\
\hline I & 3 & 2 & 0.128 & & & \\
\hline II & 0 & 6 & - & & & \\
\hline III & 7 & 22 & - & & & \\
\hline IV & 9 & 16 & - & & & \\
\hline \multicolumn{7}{|l|}{ Dukes' stage } \\
\hline A & & & & 3 & 3 & 0.297 \\
\hline B & & & & 1 & 10 & - \\
\hline $\mathrm{C}$ & & & & 2 & 6 & - \\
\hline $\mathrm{D}$ & & & & 2 & 9 & - \\
\hline
\end{tabular}

The Student's t-test was used to analyze age and tumor size; the Chi-square test was used to analyze categorical variables. The traditional group involved double-layer manual and stapled suture. SD, standard deviation.

Control TNM classification for gastric cancer and the Dukes' classification for rectal cancer.

Following the surgery, blood samples were collected and routine blood, liver and renal function tests were performed.

Anastomotic complications. Radiological assessment of the anastomotic integrity for the esophagogastric and colorectal anastomoses was performed using a water-soluble contrast enema 4-14 days postoperatively. Any extravasation of the contrast medium detected on radiography was considered a radiological leak. A clinical leak was defined as the appearance of fecal material in the abdominal drains, development of a colocutaneous fistula, or the development of systemic sepsis associated with local peritoneal signs during the postoperative period. Clinically significant anastomotic strictures were defined as those requiring surgical dilation in patients who developed symptoms of outlet obstruction.

QOL. The European Organization for Research and Treatment of Cancer (EORTC) QLQ-C30 patient questionnaire was used, which is a recognized reliable and validated QOL evaluation tool $(25,26)$. The questionnaire combined 12 questions for a global QOL and symptom assessment, including pain, fatigue, diarrhea and constipation. At the time of the primary treatment, the surgeons requested informed consent from the patients to receive a QOL survey. Questionnaires were subsequently sent to the willing participants and collected within 6 months postoperatively. The mean follow-up period was $2.7 \pm 0.3$ months.

Statistical methods. The data were analyzed using SPSS software, version 11.5 (SPSS Inc., Chicago, IL, USA). The Student's t-test and Chi-square test were used to analyze continual and categorical variables, respectively. To elucidate the risk factors for postoperative complications, a multivariate analysis was performed using the logistic regression model.

\section{Results}

Characteristics of patients in the new manual and traditional groups. Table I summarizes the characteristics of the 
Table II. Comparison of preoperative data between patients undergoing anastomosis with the new manual and traditional techniques.

\begin{tabular}{lcc}
\hline Variables & New manual (mean \pm SD) & Traditional (mean \pm SD) \\
\hline Surgical time (h) & $4.58 \pm 1.04$ & $4.57 \pm 1.47$ \\
Intraoperative blood loss (ml) & $215.78 \pm 141.47$ & $262.50 \pm 182.27$ \\
Time until regular diet tolerated (days) & $6.31 \pm 1.57$ & $7.00 \pm 1.71$ \\
Time until first bowel movement (days) & $4.88 \pm 1.07$ & $5.24 \pm 1.32$ \\
Abdominal drainage (ml) & & 0.343 \\
Day 1 & $126.32 \pm 81.77$ & $208.88 \pm 182.24$ \\
Day 2 & $59.42 \pm 62.39$ & $93.70 \pm 114.76$ \\
Day 3 & $29.85 \pm 42.42$ & $84.84 \pm 110.67$ \\
Day 7 & $15.38 \pm 36.66$ & $45.70 \pm 94.5$ \\
Temperature ( ${ }^{\circ}$ C) & $37.79 \pm 0.70$ & $37.58 \pm 0.47$ \\
Blood transfusion (ml) & $274.07 \pm 419.33$ & 0.013 \\
Expenditure of surgery (RMB) & $3,509.85 \pm 768.68$ & 0.036 \\
Hospital stay (days) & $14.60 \pm 4.19$ & 0.005 \\
\hline
\end{tabular}

${ }^{a}$ The value was obtained from the group undergoing staple suture. The Student's t-test was used to analyze all the variables. The traditional group involved double-layer manual and stapled suture. SD, standard deviation; RMB, renminbi.

Table III. Pre- and postoperative routine blood and liver function tests in patients undergoing anastomosis with the new manual and traditional techniques.

\begin{tabular}{|c|c|c|c|c|c|c|}
\hline \multirow[b]{2}{*}{ Variables } & \multicolumn{2}{|c|}{ Preoperative } & \multirow[b]{2}{*}{ P-value } & \multicolumn{2}{|c|}{ Postoperative } & \multirow[b]{2}{*}{ P-value } \\
\hline & $\begin{array}{l}\text { New manual } \\
(\text { mean } \pm S D)\end{array}$ & $\begin{array}{c}\text { Traditional } \\
(\text { mean } \pm \mathrm{SD})\end{array}$ & & $\begin{array}{l}\text { New manual } \\
(\text { mean } \pm \mathrm{SD})\end{array}$ & $\begin{array}{c}\text { Traditional } \\
(\text { mean } \pm \text { SD })\end{array}$ & \\
\hline \multicolumn{7}{|l|}{ Routine blood test } \\
\hline WBC $\left(10^{9} / 1\right)$ & $5.88 \pm 2.62$ & $6.56 \pm 2.86$ & 0.415 & $9.18 \pm 3.13$ & $9.12 \pm 7.08$ & 0.966 \\
\hline GRA $(\%)$ & $62.86 \pm 15.54$ & $67.49 \pm 8.58$ & 0.264 & $75.23 \pm 8.32$ & $74.37 \pm 12.25$ & 0.797 \\
\hline $\operatorname{RBC}\left(10^{12} / 1\right)$ & $4.19 \pm 0.83$ & $3.85 \pm 0.53$ & 0.128 & $3.85 \pm 0.68$ & $3.82 \pm 0.48$ & 0.855 \\
\hline $\operatorname{HGB}(\mathrm{g} / \mathrm{l})$ & $122.44 \pm 28.60$ & $107.43 \pm 25.32$ & 0.068 & $114.67 \pm 22.65$ & $110.80 \pm 11.77$ & 0.541 \\
\hline $\operatorname{PLT}\left(10^{9} / 1\right)$ & $198.13 \pm 75.20$ & $248.74 \pm 111.73$ & 0.084 & $260.07 \pm 110.32$ & $264.86 \pm 123.01$ & 0.898 \\
\hline \multicolumn{7}{|l|}{ Liver function } \\
\hline T.BIL $(\mu \mathrm{mol} / \mathrm{l})$ & $14.12 \pm 6.89$ & $14.10 \pm 5.96$ & 0.992 & $16.23 \pm 7.80$ & $10.08 \pm 3.11$ & 0.116 \\
\hline $\operatorname{ALT}(\mathrm{U} / \mathrm{l})$ & $23.48 \pm 16.75$ & $27.31 \pm 20.14$ & 0.553 & $48.93 \pm 49.21$ & $47.33 \pm 30.86$ & 0.931 \\
\hline $\mathrm{TP}(\mathrm{g} / \mathrm{l})$ & $65.95 \pm 8.09$ & $63.92 \pm 20.99$ & 0.693 & $60.98 \pm 7.50$ & $66.04 \pm 8.66$ & 0.165 \\
\hline $\mathrm{ALB}(\mathrm{g} / \mathrm{l})$ & $39.67 \pm 4.72$ & $36.20 \pm 11.25$ & 0.210 & $33.57 \pm 4.24$ & $34.64 \pm 4.97$ & 0.560 \\
\hline \multicolumn{7}{|l|}{ Renal function } \\
\hline BUN (mmol/l) & $5.95 \pm 2.51$ & $4.75 \pm 1.47$ & 0.143 & $4.49 \pm 1.54$ & $5.67 \pm 2.31$ & 0.210 \\
\hline $\mathrm{CR}(\mu \mathrm{mol} / \mathrm{l})$ & $76.66 \pm 21.76$ & $74.75 \pm 21.84$ & 0.812 & $62.36 \pm 16.29$ & $74.00 \pm 28.51$ & 0.284 \\
\hline Glucose (mmol/l) & $5.32 \pm 1.66$ & $5.42 \pm 0.82$ & 0.872 & $6.85 \pm 2.63$ & $7.83 \pm 2.82$ & 0.654 \\
\hline \multicolumn{7}{|l|}{ Blood electrolytes } \\
\hline $\mathrm{Na}(\mathrm{mmol} / \mathrm{l})$ & $141.74 \pm 4.09$ & $140.52 \pm 2.76$ & 0.407 & $135.99 \pm 5.22$ & $138.30 \pm 2.80$ & 0.284 \\
\hline $\mathrm{K}(\mathrm{mmol} / \mathrm{l})$ & $4.03 \pm 0.48$ & $3.86 \pm 0.59$ & 0.387 & $4.10 \pm 0.71$ & $4.49 \pm 0.77$ & 0.249 \\
\hline $\mathrm{Cl}(\mathrm{mmol} / \mathrm{l})$ & $102.45 \pm 3.53$ & $101.60 \pm 2.87$ & 0.518 & $98.05 \pm 5.44$ & $98.86 \pm 2.50$ & 0.714 \\
\hline
\end{tabular}

The Student's t-test was used to assess the statistical significance of the differences in the tumor volume between the new manual and traditional groups. The traditional group involved double-layer manual and stapled suture. SD, standard deviation; WBC, white blood cell count; GRA, granulocyte; RBC, red blood cell count; HGB, hemoglobin; PLT, platelet count; T.BIL, total serum bilirubin; ALT, alanine transaminase; TP, serum protein; ALB, albumin; BUN, blood urea nitrogen; CR, creatinine; Na, sodium; K, potassium; Cl, chlorine. 
Table IV. Postoperative complications in the two groups.

\begin{tabular}{lccc}
\hline Complications & $\begin{array}{c}\text { New } \\
\text { manual }\end{array}$ & Traditional P-value \\
\hline Hospital death & 0 & 0 & - \\
Reoperation & 0 & 1 & - \\
Cardiac complications & - & 1 & - \\
Pulmonary complications & 1 & 7 & - \\
Wound infection & 1 & 7 & - \\
Severe bleeding & - & 3 & - \\
Chylous leakage & - & 1 & - \\
Anastomosis stricture & - & 1 & - \\
Anastomosis leakage & - & 2 & - \\
Any complication $(\%)$ & $2(7.40)$ & $23(31.08)$ & 0.018 \\
Anastomotic complication $(\%)$ & 0 & $3(4.05)$ & 0.288 \\
\hline
\end{tabular}

The Chi-square test was used to assess the incidence of complications. The traditional group involved double-layer manual and stapled suture.

101 patients. There were no significant differences between the groups regarding preoperative variables, such as tumor differentiation, pathological stage, pathological type, tumor size, lymph node metastasis or basic anthropometric data.

Comparison of preoperative data. The average surgical time in the new manual technique group was $4.58 \pm 1.04 \mathrm{~h}$ and in the traditional manual and staple group was $4.57 \pm 1.47 \mathrm{~h}$ $(\mathrm{P}=0.965)$. There was no significant difference in the amount of intraoperative blood loss between the two groups.

Following the surgery, there were no significant differences between the two groups with regard to the highest recorded temperature, time until a regular diet was tolerated and time until the first bowel movement. However, the volume of abdominal drainage fluid at 1,2,3 and 7 days in the new manual technique group was significantly lower compared with that in the traditional group.

The blood transfusion volume in the new manual technique group was lower compared to that in the traditional technique group (274.07 \pm 419.33 vs. $646.67 \pm 1,146.06 \mathrm{ml}$, respectively; $\mathrm{P}=0.053)$. The postoperative hospital stay was shorter in the new manual compared to that in the traditional technique group $(14.60 \pm 4.19$ vs. $17.60 \pm 6.29$ days, respectively; $\mathrm{P}=0.038)$. The total expenditure of the surgery was lower in the new manual compared to that in the staple group $(3,509.85 \pm 768.68 \mathrm{vs}$ $6,141.83 \pm 308.90$ renminbi, respectively; $\mathrm{P}=0.001$ ) (Table II).

Blood tests. There were no significant differences in the routine blood, liver and renal function tests, or in glucose and electrolyte levels between the new manual and traditional technique groups (Table III).

Complications. The incidence rate of complications in the new manual technique group was $7.40 \%$, which was statistically significantly lower compared to that in the traditional group, which was $31.08 \%(\mathrm{P}=0.018)$. However, there were no differences regarding the complications of the anastomotic
Table V.Logistic regression analysis of the association between postoperative complications and preoperative variables for the 101 patients.

\begin{tabular}{lccc}
\hline Variables & $\begin{array}{c}\text { Regression } \\
\text { coefficient }\end{array}$ & $\begin{array}{c}\text { Standard } \\
\text { error }\end{array}$ & P-value \\
\hline New manual suture & -1.69 & 0.96 & 0.078 \\
Tumor stage & 0.92 & 0.52 & 0.080 \\
Tumor grade & - & - & 0.402 \\
Age & - & - & 0.550 \\
Gender & - & - & 0.223 \\
Pathological type & - & - & 0.744 \\
Gastric/rectal cancer & - & - & 0.332 \\
Underlying disease & - & - & 0.483 \\
Surgical bleeding & - & - & 0.899 \\
\hline
\end{tabular}

The new manual suture was coded as 1 , yes, 2, no; tumor stage was coded from 1 to 4 with increasing stage; tumor grade was coded from 1 to 3 with increasing grade; gender was coded as 1 , male and 2, female; pathological type was coded as 1 , adenocarcinoma and 2, non-adenocarcinoma; underlying disease including hypertension, chronic bronchitis, diabetes mellitus and chronic hepatitis was coded as 0 , absent and 1 , present.

port ( 0 vs. $4.05 \%$; $\mathrm{P}=0.288$ ) (Table IV). To further assess the higher risk of postoperative complications, a multivariate analysis was conducted using the logistic regression model, including suture methods, tumor stage, tumor grade, age, gender, pathological type, cancer type, underlying disease and surgical bleeding. The analysis demonstrated that the new manual suture technique was negatively correlated with the total complication rate (Table V).

Comparisons of QOL between suture groups. The mean scores for all EORTC QLQ-30 questions in new manual and traditional technique groups were $18.46 \pm 6.59$ vs. $16.13 \pm 6.03$. There were no statistically significant differences between the two groups $(\mathrm{P}=0.31)$ (Table VI).

\section{Discussion}

In this study, we used the continuous single-layer suture in the posterior wall of the anastomosis of the digestive tract in 27 cases involving difficult surgical locations and demonstrated that this new manual anastomostic method is technically possible to perform and appears to be as efficient and safe as the traditional and stapling techniques. The complication rate was the same or lower compared to that observed with stapling and traditional anastomotic techniques. Moreover, the overall expenditure was significantly reduced.

Our results demonstrated that the total complication rate in the new manual technique group was $7.4 \%$ and the anastomotic complication rate was zero, which was lower than that recorded in the traditional technique group and previous studies (4-8). The improved results of our study may be attributed to the 4 main advantages of the new manual method: i) improved exposure; ii) the continuous suture is tighter compared to 
Table VI. Evaluation of QOL scores in the two groups.

\begin{tabular}{lccc}
\hline Symptoms & New manual (mean $\pm \mathrm{SD}$ ) & Traditional (mean $\pm \mathrm{SD})$ & P-value \\
\hline Fatigue & $1.55 \pm 1.04$ & $1.46 \pm 0.93$ & 0.806 \\
Sleep & $1.55 \pm 0.82$ & $1.08 \pm 0.41$ & 0.032 \\
Pain & $1.73 \pm 1.27$ & $1.71 \pm 1.27$ & 0.968 \\
Constipation & $1.18 \pm 0.40$ & $1.00 \pm 0.00$ & 0.032 \\
Diarrhea & $1.27 \pm 0.65$ & $1.38 \pm 0.88$ & 0.732 \\
Micturition problems & $1.09 \pm 0.30$ & $1.00 \pm 0.00$ & 0.142 \\
Gastrointestinal problems & $2.18 \pm 1.25$ & $1.83 \pm 1.24$ & 0.447 \\
Defecation problems & $1.45 \pm 0.69$ & $1.04 \pm 0.20$ & 0.010 \\
Nausea/vomiting & $1.73 \pm 1.27$ & $1.38 \pm 1.01$ & 0.385 \\
Loss of appetite & $2.18 \pm 1.33$ & $1.75 \pm 1.19$ & 0.343 \\
Weight loss & $1.55 \pm 0.93$ & $1.46 \pm 0.93$ & 0.799 \\
Dyspnea & $1.00 \pm 0.00$ & $1.04 \pm 0.20$ & 0.507 \\
Sum & $18.45 \pm 6.59$ & $16.13 \pm 6.03$ & 0.310 \\
Follow-up time (months) & $2.73 \pm 1.89$ & $2.46 \pm 1.63$ & 0.669 \\
\hline
\end{tabular}

The Student's t-test was used to analyze all the variables. The traditional group involved double-layer manual and stapled suture. SD, standard deviation; QOL, quality of life.

the interrupted suture; iii) less tissue is turned inwards; and iv) less tissue reaction to the prolene thread.

The difficulty of the anastomosis lies with limited exposure, particularly of the posterior wall, in certain anatomical locations. The anastomosis may not be adequately visualized due to the surrounding tissues; even with the use of surgical staplers, the procedure is performed blindly. However, the continuous suture does not require a prior butt joint, which makes the exposure of the posterior wall easier and contributes to convenience and safety.

The first basic consideration regarding the surgical technique of gastrointestinal anastomosis is mechanical integrity. Undoubtedly, among various types of anastomosis, the continuous suture is the tightest, which explains the fact that it is commonly used for blood vessel sutures. Furthermore, it was demonstrated that single-layer anastomosis is as strong as double-layer suturing in the small intestine and colon and ensures mechanical integrity (24). The second consideration is tissue viability, which is closely associated with adequate blood supply and good nutritional status of the suture line $(16,18)$. Blood flow is always reduced in the suture line compared with the normal mucosa. Of all the anastomoses studied, the blood flow of the suture line decreased from the single-layered manual to the two-layered manual to the stapled suture $(17,18)$.

It was previously demonstrated that the surgical time may be reduced by $\leq 30 \mathrm{~min}$ for each stapled anastomosis (27) and the continuous suture may also contribute to the reduction in surgical time without increasing the complication rate in various types of surgery (28). There was no significant difference in surgical time between the new manual and traditional suture technique in this study. Staple anastomosis may help reduce surgery time; however, to ensure a secure anastomosis, the use of sharp blade is critical and the sectioned tissues must be handled with caution to avoid intraoperative problems, which may ultimately prolong surgery time.
The development of strictures is closely associated with the diameter of the anastomosis and the thickness of the wall. Staple and conventional manual sutures are double-layer sutures and, in certain cases, a strengthening suture may be required following stapling. The staple anastomosis 'reinforced' with sutures is a three-layered affair, leading to more tissue turning inwards and inducing stricture formation (18). Staplers of different external diameters are associated with differences in the diameter of the resection site and the surface area of dissection (29). However, the precise selection of a stapler diameter is almost impossible, due to the fixed available types. By contrast, single-layer suturing causes less tissue to turn inwards, which may reduce the incidence of anastomotic strictures. In addition, the continuous suture was proven to contribute to the adjustment of the anastomostic diameter (30).

In conclusion, our results suggest that single-layer continuous suture in the posterior wall of the anastomosis of the digestive tract is a novel, feasible and safe method that may simplify the surgical procedure in anastomoses that present with difficulty due to special anatomical location, while reducing overall expenditure.

\section{Acknowledgements}

This study was supported by the Science Research and Technology Development Program of Shaanxi (grant no. 2010K15-07-03). The authors would like to thank Dr An Xin-Ming and Wang Na for their help with data collection and statistical analysis.

\section{References}

1. Ferlay J, Shin HR, Bray F, Forman D, Mathers C and Parkin DM: Estimates of worldwide burden of cancer in 2008: GLOBOCAN 2008. Int J Cancer 127: 2893-2917, 2010. 
2. Weeks JC, Nelson H, Gelber S, et al: Short-term quality-of-life outcomes following laparoscopic-assisted colectomy vs. open colectomy for colon cancer: a randomized trial. JAMA 287 321-328, 2002

3. Briel JW, Tamhankar AP, Hagen JA, et al: Prevalence and risk factors for ischemia, leak, and stricture of esophageal anastomosis: gastric pull-up versus colon interposition. J Am Coll Surg 198: 536-542, 2004

4. No authors listed: Suturing or stapling in gastrointestinal surgery: a prospective randomized study. West of Scotland and Highland Anastomosis Study Group. Br J Surg 78: 337-341, 1991.

5. Laterza E, de' Manzoni G, Veraldi GF, Guglielmi A, Tedesco P and Cordiano C: Manual compared with mechanical cervical oesophagogastric anastomosis: a randomised trial. Eur J Surg 165: 1051-1054, 1999.

6. Law S, Fok M, Chu KM and Wong J: Comparison of hand-sewn and stapled esophagogastric anastomosis after esophageal resection for cancer: a prospective randomized controlled trial. Ann Surg 226: 169-173, 1997.

7. Okuyama M, Motoyama S, Suzuki H, Saito R, Maruyama K and Ogawa J: Hand-sewn cervical anastomosis versus stapled intrathoracic anastomosis after esophagectomy for middle or lower thoracic esophageal cancer: a prospective randomized controlled study. Surg Today 37: 947-952, 2007.

8. Walther B, Johansson J, Johnsson F, Von Holstein CS and Zilling T: Cervical or thoracic anastomosis after esophageal resection and gastric tube reconstruction: a prospective randomized trial comparing sutured neck anastomosis with stapled intrathoracic anastomosis. Ann Surg 238: 803-814, 2003

9. Kuroyanagi H, Oya M, Ueno M, Fujimoto Y, Yamaguchi T and Muto T: Standardized technique of laparoscopic intracorporeal rectal transaction and anastomosis for low anterior resection. Surg Endosc 22: 557-561, 2008.

10. Barlehner E, Benhidjeb T, Anders S and Schicke B: Laparoscopic resection for rectal cancer: outcomes in 194 patinents and review of the literature. Surg Endosc 19: 757-766, 2005.

11. Scheidbach H, Schneider C, Konradt J, et al: Laparoscopic abdominoperineal resection and anterior resection with curative intent for carcinoma of the rectum. Surg Endosc 16: 7-13, 2002.

12. Karanjia ND, Corder AP, Holdsworth PJ and Heald RJ: Risk of peritonitis and fatal septicaemia and the need to defunction the low anastomosis. Br J Surg 78: 196-198, 1991.

13. Ziv Y, Fazio VW, Church JM, Lavery IC, King TM and Ambrosetti P: Stapled ileal pouch anal anastomoses are safer than handsewn anastomoses in patients with ulcerative colitis. Am J Surg 171: 320-323, 1996.

14. Orringer MB, Marshall B and Iannettoni MD: Eliminating the cervical esophagogastric anastomotic leak with a side-to-side stapled anastomosis. J Thorac Cardiovasc Surg 119: 277-288, 2000.

15. Docherty JG, McGregor JR, Akyol AM, Murray GD and Galloway DJ: Comparison of manually constructed and stapled anastomoses in colorectal surgery. West of Scotland and Highland Anastomosis Study Group. Ann Surg 221: 176-184, 1995.
16. Markar SR, Karthikesalingam A, Vyas S, Hashemi M and Winslet M: Hand-sewn versus stapled oesophago-gastric anastomosis: systematic review and meta-analysis. J Gastrointest Surg 15: 876-884, 2011

17. Orsay CP, Bass EM, Firfer B, Ramakrishnan V and Abcarian $\mathrm{H}$ Blood flow in colon anastomotic stricture formation. Dis Colon Rectum 38: 202-206, 1995.

18. Chung RS: Blood flow in colonic anastomoses. Effect of stapling and suturing. Ann Surg 206: 335-339, 1987.

19. Wong J, Cheung H, Lui R, Fan YW, Smith A and Siu KF: Esophagogastric anastomosis performed with a stapler: the occurrence of leakage and stricture. Surgery 101: 408-415, 1987.

20. Berrisford RG, Page RD and Donnelly RJ: Stapler design and strictures at the esophagogastric anastomosis. J Thorac Cardiovasc Surg 111: 142-146, 1996

21. Valdivieso A, Sarabia S, Pocino R, et al: Is it worth using mechanical sutures in gastric surgery? Acta Chir Belg 95 (Suppl 4): 179-181, 1995

22. Dziki AJ, Duncan MD, Harmon JW, et al: Advantages of handsewn over stapled bowel anastomosis. Dis Colon Rectum 34: 442-448, 1991

23. Fok M, Ah-Chong AK, Cheng SW and Wong J: Comparison of a single layer continuous hand-sewn method and circular stapling in 580 oesophageal anastomoses. Br J Surg 78: 342-345, 1991

24. Valverde A, Hay JM, Fingerhut A and Elhadad A: Manual versus mechanical esophagogastric anastomosis after resection for carcinoma: a controlled trial. French Associations for Surgical Research. Surgery 120: 476-483, 1996.

25. Aaronson NK, Ahmedzai S, Bergman B, et al: The European Organization for Research and Treatment of Cancer QLQ-C30: a quality-of-life instrument for use in international clinical trials in oncology. J Natl Cancer Inst 85: 365-376, 1993.

26. Engel J, Kerr J, Schlesinger-Raab A, Eckel R, Sauer H and Holzel D: Quality of life in rectal cancer patients: a four-year prospective study. Ann Surg 238: 203-213, 2003.

27. Shoji Y, Nihei Z, Hirayama R and Mishima Y: Experiences with the linear cutter technique for performing Roux-en-Y anastomosis following total gastrectomy. Surg Today 25: 27-31, 1995.

28. Valenzuela P, Saiz Puente MS, Valero JL, Azorin R, Ortega R and Guijarro R: Continuous versus interrupted sutures for repair of episiotomy or second-degree perineal tears: a randomised controlled trial. BJOG 116: 436-441, 2009.

29. Hirahara N, Monma H, Shimojo Y, et al: Reconstruction of the esophagojejunostomy by double stapling method using $\mathrm{EEA}^{\mathrm{TM}} \mathrm{OrVil}^{\mathrm{TM}}$ in laparoscopic total gastrectomy and proximal gastrectomy. World J Surg Oncol 9: 55, 2011.

30. Park KJ, Woo JS, Jeong SS and Yi JH: Continuous 'over and over' suture for tricuspid ring annuloplasty. Korean J Thorac Cardiovasc Surg 45: 19-23, 2012. 